\title{
Mass Spectrometry
}

National Cancer Institute

\section{Source}

National Cancer Institute. Mass Spectrometry. NCI Thesaurus. Code C17156.

An analytical technique wherein ions are separated according to their ratio of charge to

mass. From the mass spectrum produced, the atomic weight of the particle can be deduced. 